\title{
Shaping functional architecture by oscillatory alpha activity: gating by inhibition
}

\section{Ole Jensen* and Ali Mazaheri}

Donders Institute for Brain, Cognition and Behavior, Radboud University, Nijmegen, Netherlands

\section{Edited by:}

Thilo Womelsdorf, Robarts Research Institute London, Canada

Reviewed by:

Tobias H. Donner, University of

Amsterdam, Netherlands

Stephanie R. Jones, Massachusetts

General Hospital, USA

${ }^{*}$ Correspondence:

Ole Jensen, Donders Institute for Brain, Cognition and Behaviour, Centre

for Cognitive Neuroimaging, P.O. Box

9101, NL-6500 HB Nijmegen,

Netherlands.

e-mail: ole.jensen@donders.ru.nl
In order to understand the working brain as a network, it is essential to identify the mechanisms by which information is gated between regions. We here propose that information is gated by inhibiting task-irrelevant regions, thus routing information to task-relevant regions. The functional inhibition is reflected in oscillatory activity in the alpha band (8-13 Hz). From a physiological perspective the alpha activity provides pulsed inhibition reducing the processing capabilities of a given area. Active processing in the engaged areas is reflected by neuronal synchronization in the gamma band $(30-100 \mathrm{~Hz})$ accompanied by an alpha band decrease. According to this framework the brain could be studied as a network by investigating crossfrequency interactions between gamma and alpha activity. Specifically the framework predicts that optimal task performance will correlate with alpha activity in task-irrelevant areas. In this review we will discuss the empirical support for this framework. Given that alpha activity is by far the strongest signal recorded by EEG and MEG, we propose that a major part of the electrophysiological activity detected from the working brain reflects gating by inhibition.

Keywords: magnetoencephalography, electroencephalography, alpha, gamma, functional connectivity, effective connectivity

\section{HYPOTHESIS: GATING BY INHIBITION}

The human brain is a complex processing device which rapidly adapts to the environment. This requires fast changes in the functional architecture of the brain network which must rely on dynamic interactions. Network questions are often addressed by asking how different regions become functionally coupled depending on the task at hand (Friston et al., 1997). This can be conceptualized as the question of how information is gated from a sending region to one of two receiving regions. This could be achieved by fast changes in synaptic strength, for instance due to changes in presynaptic vesicle release (Figure 1A). It has also been proposed that functional connectivity between task-relevant regions is established by oscillatory phase coupling between the sending and receiving region (Bressler, 1995; Varela et al., 2001; Fries, 2005) (Figure 1B). We here promote the idea that information is routed by functionally blocking off the task-irrelevant pathways: gating by inhibition (Figure 1C). Importantly, this inhibition is reflected by oscillatory activity in the alpha band. This functional inhibition might be a consequence of GABAergic inhibition provided by the interneuronal network. We will later address this issue in detail. Nevertheless, we propose that the alpha activity is a consequence pulsed inhibition which reduces the processing capabilities of a given area.

\section{ALPHA OSCILLATIONS REFLECT FUNCTIONAL INHIBITION}

Oscillatory activity in the alpha and beta bands was first discovered in the late 1920s by Hans Berger and is by far the strongest electrophysiological signal measured from the human waking brain (Berger, 1929; da Silva et al., 1973; Niedermeyer and Lopes da Silva, 1999). Alpha activity has for a long period been interpreted to reflect "cortical idling" (Pfurtscheller et al., 1996). This interpretation was based on the finding that alpha activity increases when subjects are awake but not engaged in any task. Recently strong evidence has accumulated against the idling hypothesis and it is becoming clear that the functional role of alpha activity has been highly underappreciated.

The recent insight into the functional role of alpha activity has been brought about by high-density EEG and MEG recordings. The improved spatial resolution of these techniques has facilitated the functional interpretation of the measured activity. One strong piece of evidence demonstrating that alpha activity cannot reflect idling, stems from working memory experiments in which subjects were presented items to be remembered for a shorter period. Using these types of paradigms several studies have now demonstrated a robust increase in alpha power with memory load during the retention interval (Jensen et al., 2002; Tuladhar et al., 2007; Scheeringa et al., 2009). The alpha increase has been suggested to either reflect active processing related to memory maintenance (Palva and Palva, 2007; Palva et al., 2007) or inhibition of posterior regions not required for the task (Klimesch et al., 2007).

In order to conclusively determine if the alpha activity is reflecting functional inhibition, experiments are required which can tell whether the alpha activity is related to the engagement or disengagement of specific brain regions. For instance it has been demonstrated that alpha activity over visual areas increases in motor tasks and vice versa (Pfurtscheller, 1992). Studies of spatial attention have shown that when covert attention is directed to one hemifield (e.g., the left), then alpha decreases in the contralateral (right) but increases in the ipsilateral (left) hemisphere (Worden et al., 2000; Thut et al., 2006; Rihs et al., 2007; Kelly et al., 2009; van Gerven and 


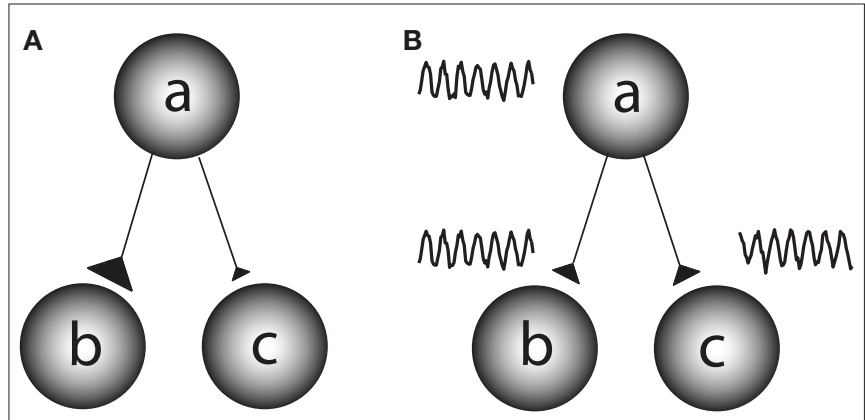

C

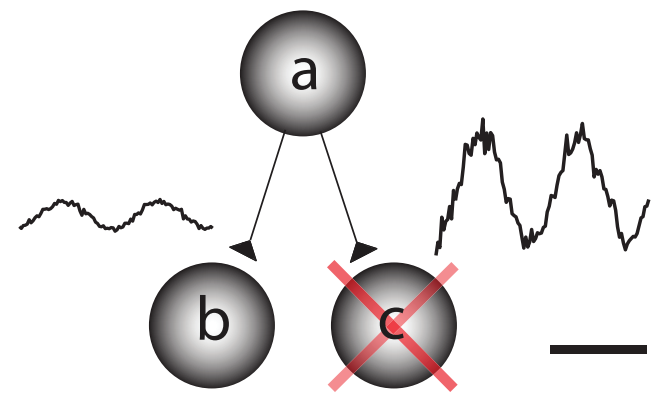

FIGURE 1 | Different principles by which information can be gated through a network. Consider a situation in which information is supposed to be routed from node $\mathbf{a}$ to node $\mathbf{b}$ but not from node $\mathbf{a}$ to node $\mathbf{c}$. (A) One possibility is that the synaptic connections from node $\mathbf{a}$ to $\mathbf{b}$ are strengthened on a fast time scale and weakened from node $\mathbf{a}$ to $\mathbf{c}$. This would require a mechanism for synaptic plasticity that works on a fast time scale. (B) Information might be gated through neuronal phase-synchronization between node $\mathbf{a}$ and $\mathbf{c}$. The information flow from node $\mathbf{b}$ to $\mathbf{c}$ is blocked by adjusting the phase difference. (C) We here promote the principle of gating by inhibition. Node $\mathbf{c}$ is actively suppressed by functional inhibition. This serves to gate the information flow from $\mathbf{a}$ to $\mathbf{b}$. The functional inhibition is reflected in the 9-13 Hz alpha band.

Jensen, 2009; Handel et al., 2010). Interestingly, this hemispheric alpha lateralization had consequences for detection performance even when it was attenuated by moving-dot kinematograms (Handel et al., 2010). The modulation of the posterior alpha activity with covert attention is so robust even in single trials that it can be used as a control signal for brain-computer interfaces (van Gerven and Jensen, 2009; Bahramisharif et al., 2010). A similar pattern was observed in working memory tasks in which subjects were presented visual stimuli to be remembered in either the left or right visual hemifield (Van Der Werf et al., 2008; Grimault et al., 2009; Sauseng et al., 2009). In a cross-modal EEG study subjects were asked to attend either to visual or auditory stimuli (Fu et al., 2001). The posterior alpha activity increased only when subjects attended to the auditory stimuli. The suppressive nature of alpha is not only present in visuo-spatial paradigms. In a recent MEG study subjects were asked to maintain either the identity or orientation of presented faces (Jokisch and Jensen, 2007). A clear increase in alpha activity over the dorsal stream was observed when the ventral stream was selectively engaged by the face identity task (Figure 2). In a somatosensory working memory task, the alpha activity decreased in the primary sensorimotor cortex contralateral to the engaged hand while it increased in the ipsilateral hemisphere (Haegens et al., 2010). The functional role of alpha activity in defining the brain state has also been observed in auditory studies. A recent study found that alpha power at parieto-occipital sites was lateralized by the direction of auditory attention to continuous speech (Kerlin et al., 2010). The alpha lateralization might serve to engage the processing of visual input associated with auditory input from the same hemifield. In a working memory study on maintaining pitches, alpha activity from left superior temporal areas increased during the retention interval (van Dijk et al., 2010). This temporal alpha activity, possibly being the tau-rhythm (Lehtela et al., 1997), likely reflected inhibition of the left auditory cortex to allocate resources to the right auditory cortex involved in pitch processing. In sum, these studies make the case that alpha activity decreases in engaged regions, while it increases in disengaged regions.

\section{IS FUNCTIONAL INHIBITION BY ALPHA ACTIVITY IN TASK-IRRELEVANT AREAS REQUIRED FOR OPTIMAL TASK PERFORMANCE?}

In some of our recent studies we have been able to demonstrate cases where the increase in alpha activity in task-irrelevant areas correlates with task performance. This suggests that alpha activity in task-irrelevant regions is required for optimal performance. Such an effect was first shown in a somatosensory working memory task in which stimuli were delivered to the right hand (Haegens et al., 2010). This resulted in alpha power increases over the task-irrelevant right somatosensory areas during the retention interval (Figure 3). Furthermore, this increase correlated with performance, i.e., more errors were made when the right hemisphere alpha activity was not sufficiently strong. Interestingly, the alpha decrease in the left hemisphere did not correlate with performance; nor did the left hemisphere gamma increase. This effect is not specific to working memory tasks. Recently we conducted a long-term memory experiment in which subjects were asked to learn sets of lists three words long. We then characterized the brain activity during encoding with respect to lists that were later remembered and forgotten. We found a dramatic increase in alpha activity over occipital regions for later remembered lists (Meeuwissen et al., in press). Interestingly, it was possible to predict if a given list was later remembered on a single trial basis from the alpha activity alone. Given that the occipital regions are not required for the task, we suggest that successful long-term memory encoding is improved by active inhibition of occipital regions. Importantly, the strongest subsequent memory effect was found in the alpha band when compared to effects in the gamma band. These two studies show that performance is impaired if functional inhibition reflected by alpha band activity fails in taskirrelevant regions. This is consistent with the notion that optimal task performance is dependent on shutting down task-irrelevant regions in order to allocate resources to talk-relevant regions.

\section{PRE-STIMULUS OSCILLATORY ACTIVITY MODULATES SUBSEOUENT PROCESSING}

It is becoming increasingly clear that ongoing oscillatory activity prior to an event has a strong impact on subsequent processing. Indeed it has been demonstrated that posterior pre-stimulus alpha 
A

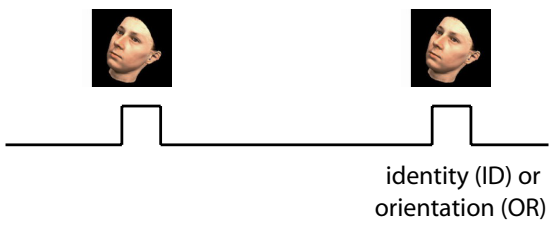

B

B ID

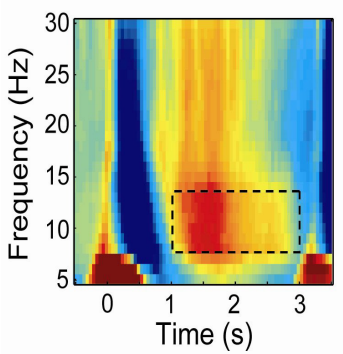

C
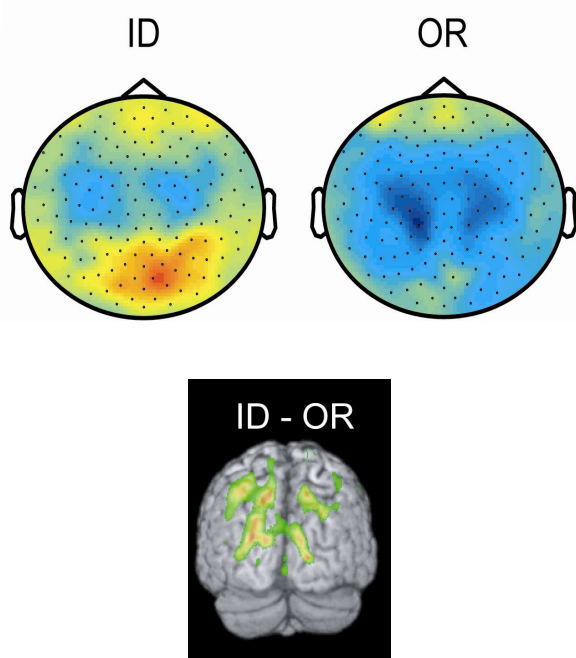

FIGURE 2 | Posterior alpha activity increases when the ventral stream, but not the dorsal stream, is engaged. (A) In a delayed-match-to-sample task subjects were asked to either maintained the identity (ID) or orientation (OR) of a presented face. (B) Time-frequency representations of power of the MEG signal over posterior regions measured during the task. Alpha activity increased in the ID task engaging the ventral stream, but remained low in the OR task engaging the dorsal stream. (C) The topographies of the alpha power showed that the increase in the ID condition was stronger over posterior central regions. (D) A beamforming analysis identified the alpha increase to parieto-occipital areas. The findings support the notion that the dorsal stream is suppressed by alpha activity when the ventral stream is engaged. Figure adapted from Jokisch and Jensen (2007).

power modulates both perception of visual and somatosensory stimuli (Linkenkaer-Hansen et al., 2004; Babiloni et al., 2006; Hanslmayr et al., 2007; van Dijk et al., 2008; Zhang et al., 2008; Kelly et al., 2009; Mazaheri et al., 2009; Schubert et al., 2009). These findings extend beyond perceptual tasks. Recently, we demonstrated that errors made in a Go-noGo task were predicted by posterior and central alpha activity (Mazaheri et al., 2009). Interestingly, after an error was made, the posterior alpha activity was reduced as if to prevent subsequent errors. This is related to a recent study in which visuo-motor

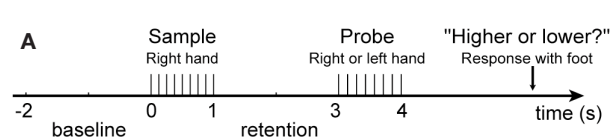

B

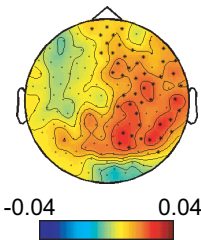

power (log ratio correct vs incorrect)
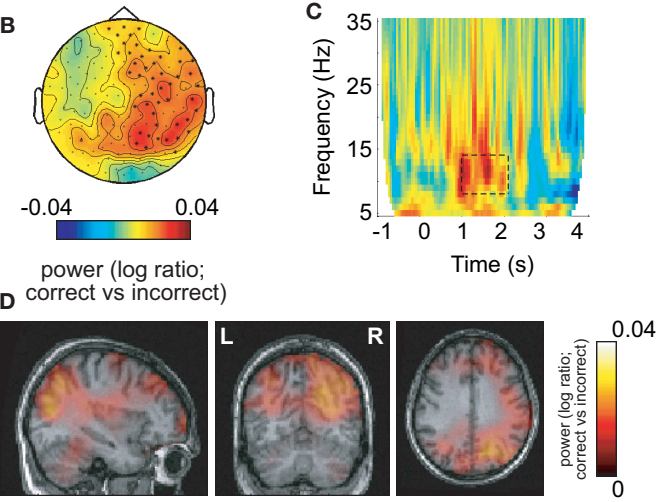

FIGURE 3 |Alpha activity in non-engaged sensorimotor areas predicts performance in a somatosensory working memory task. (A) Sample patterns of electrical stimuli were presented to the right hand. After a 2-s retention period subjects had to answer whether the frequency of the probe stimulus was higher or lower. (B) The $8-13 \mathrm{~Hz}$ alpha activity in the retention interval was compared with respect to correct and incorrect responses. The right hemisphere alpha activity was stronger for successful performance. (C) Time-frequency representations of power showed that the task-dependent effect was constrained to the alpha band in the retention interval. (D) The sources of the task-dependent alpha activity were localized in right sensorimotor and parieto-occipital regions. Reproduced from Haegens et al. (2009).

performance has been shown to be predicted by alpha activity in posterior and sensorimotor regions (Del Percio et al., 2009). Studies combining TMS and EEG have confirmed the functional inhibitory role of pre-stimulus oscillatory alpha activity (Thut and Miniussi, 2009). For instance, the group of Thut et al. has demonstrated that phosphenes evoked by TMS pulses are depressed by strong alpha activity (Romei et al., 2008; Thut and Miniussi, 2009).

While the studies mentioned above suggest a purely inhibitory role of the alpha activity, there are also studies pointing a parabolic (“inverted-U”) relationship. Linkenkaer-Hansen et al. (2004) demonstrated that weak somatosensory stimuli were best detected at medium levels of the $10 \mathrm{~Hz}$ sensorimotor rhythm (but see Schubert et al., 2009). This finding was later complemented by the demonstration that the magnitude of the somatosensory $\mathrm{N} 1$ also is related to the $10 \mathrm{~Hz}$ sensorimotor rhythm according to a parabolic relationship (Zhang and Ding, 2010). A similar relationship has been demonstrated between the posterior alpha activity and visual evoked responses (Rajagovindan and Ding, 2010). In sum, these studies suggest that pre-stimulus alpha activity is likely to be involved in shaping the functional architecture of the brain network when preparing for upcoming processing.

\section{PULSED INHIBITION}

It has been proposed that oscillatory alpha activity operates in a phasic manner (Varela et al., 1981; VanRullen and Koch, 2003), and it has been demonstrated that the phase of pre-stimulus alpha oscillations modulates visual detection (Busch et al., 2009; Mathewson et al., 
2009). On certain phases of the alpha cycle visual detection ability was high, whereas it was lower at other phases. Taken together with the inhibitory role of the alpha activity, this supports the notion of "pulsed inhibition," namely that the alpha activity produces bouts of inhibition repeated every $100 \mathrm{~ms}$ (Mazaheri and Jensen, 2010). This is consistent with a recent studies demonstrating that the gamma activity is modulated by alpha phase (Osipova et al., 2008; Voytek et al., 2010). This led us to propose that an increase in alpha activity is a consequence of an increase in the magnitude of inhibitory bouts, which serve to break the ongoing gamma activity. While it remains to be tested, it is possible that rhythmic GABAergic input from the interneuronal network is a key mechanism for producing the pulsed inhibition. The stronger the alpha activity, the shorter the "duty-cycle" of gamma activity (Figure 4). By this principle the alpha activity can provide graded inhibition by blocking the ongoing gamma activity in a phasic manner: If the alpha activity is strong, the time-window of processing is short (the duty-cycle). As alpha activity decreases in magnitude, the time-windows become longer.

How might the alpha activity serve to provide pulsed inhibition from a physiological perspective? GABAergic feedback from interneurons has been strongly implicated in the physiological mechanism generating the alpha rhythm (Jones et al., 2000; Lorincz et al., 2009). It should be mentioned that GABAergic interneurons also have been strongly implicated in the generation of the much faster 30-100 Hz gamma rhythm (Traub et al., 1999; Bartos et al., 2007; Mann and Paulsen, 2007; Cardin et al., 2009; Sohal et al., 2009). However, it is conceivable that GABAergic interneurons can be involved in producing rhythms at different temporal scales. For instance, the mechanism for the alpha activity proposed by Jones et al. (2000) relies amongst others on low-threshold T-type voltage-gated calcium channels. This type of channel produces a current with a dynamics slow enough to support the alpha generation. Interestingly, a class of somatostatin-expressing inhibitory interneurons have been identified which can produce persistent oscillations at $3-10 \mathrm{~Hz}$. These cells were identified in layer $2 / 3$ of the mouse somatosensory cortex (Fanselow et al., 2008). The GABAergic feedback could serve to directly silence processing

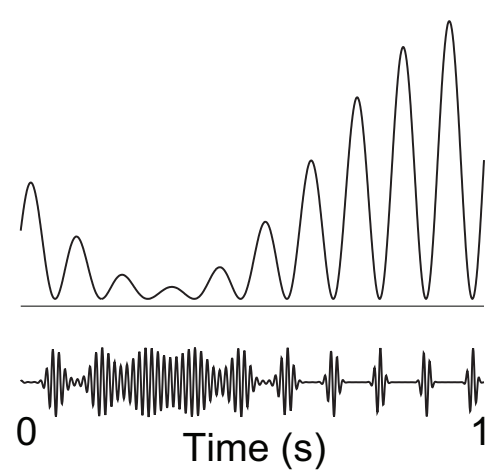

FIGURE 4 | We hypothesize that alpha activity serves to functionally disengage a given region by means of "pulsed inhibition." Consider the ongoing gamma rhythm in the lower trace which reflects neuronal processing. The pulsed alpha inhibition temporarily silences the gamma activity. As alpha goes stronger, the "duty-cycle" of gamma and thus neuronal computation decreases gradually. Reproduced from Osipova et al. (2008). in pyramidal neurons or reducing the efficacy of excitatory input by shunting inhibition (Mann and Paulsen, 2007). While mechanism have been identified for how oscillatory activity in the alpha band can be produced alone, the alpha rhythm is likely to be modulated by thalamic rhythm generators (da Silva et al., 1973; Suffczynski et al., 2001; Hughes and Crunelli, 2005; Jones et al., 2009; Lorincz et al., 2009). This drive could be implemented through phasic inhibition.

It should be mentioned that pulsed inhibition is consistent with the notion of "amplitude asymmetry." Amplitude asymmetry refers non-sinusoidal properties of the ongoing oscillatory activity resulting in the mean of the signal being biased by its magnitude (Nikulin et al., 2007; Mazaheri and Jensen, 2008; Mazaheri and Jensen, 2010). This is a consequence of the peaks of the oscillations being modulated over time rather than the troughs, i.e., it can be conceived of as rhythmic pulsing. Importantly, systematic modulation of the alpha activity can create slow event-related responses. It was recently demonstrated that such a mechanism can account for slow visual evoked responses and the sustained evoked responses observed in a working memory task (Mazaheri and Jensen, 2008; van Dijk et al., 2010). Thus the notion of pulsed inhibition holds the promise of providing insight into the physiological substrate of certain types of slow evoked responses. This is extensively discussed in the review (Mazaheri and Jensen, 2010).

\section{GAMMA ACTIVITY REFLECTS NEURONAL PROCESSING}

Numerous studies, including research on attention, working memory, long-term memory, language and motor tasks suggest that activity in the gamma range reflects engagement/processing. This was shown by intracranial, EEG and MEG recordings in animals and humans (Paller et al., 1987; Kaiser and Lutzenberger, 2005; Crone et al., 2006; Fries et al., 2007; Jensen et al., 2007; Womelsdorf and Fries, 2007; Jerbi et al., 2009). Typically gamma increases are accompanied by an alpha decrease. Since there is a strong consensus on this point, we will here only provide one of the key argument for why neuronal synchronization in the gamma band is bound to be involved in neuronal communication (Konig et al., 1996; Fries et al., 2007). Consider a group of excitatory neurons projecting to a down-stream area. Given that the time course of excitatory postsynaptic events is $10-15 \mathrm{~ms}$, these neurons will have a strong impact on the down-stream area if they synchronize at a rate of $60-100 \mathrm{~Hz}$. This implies that excitatory neuronal firing synchronized in the gamma band will be communicated to other regions. Note, that while GABAergic inhibitory interneurons are likely to be involved in setting up the gamma band synchronization, this does not equate functional inhibition. Rather, gamma band activity seems to reflect neuronal processing and communication between regions.

\section{CROSS-FREQUENCY INTERACTIONS MUST BE ASSESSED TO UNDERSTAND THE WORKING BRAIN AS A NETWORK}

In order to study the working brain as a network it is crucial to understand not only how the task-relevant regions are engaged but also how the task-irrelevant regions are inhibited. Since these processes are reflected in different frequency bands, cross-frequency interactions must be considered. There are several principles for cross-frequency interactions (Jensen and Colgin, 2007). On possibility is phase-to-phase coupling (Palva et al., 2005), e.g., the phase 
of gamma activity in one regions is coupled to the phase of the alpha activity in another (n:m coupling, e.g., four gamma cycle per alpha cycle). Another possibility is phase-to-power coupling. For instance, it was found that the gamma activity in CA1 is coupled to the phase of the theta activity in the entorhinal cortex (Colgin et al., 2009). Finally, power-to-power coupling remains a viable possibility (Bruns et al., 2000). Power-to-power correlations might reflect coupling over longer distances where the timing accuracy required for phase coupling is difficult to maintain. It should be mentioned that these measures of cross-frequency couplings are not mutually exclusive, e.g., a phase-to-power correlations might be reflected in power-to-power correlations as well. Recent MEG studies have demonstrated robust cross-frequency power-to-power coupling. One example showed that occipital gamma activity correlates negatively on a trial-by-trial basis with beta suppression in motor cortical areas during a mental rotation task of hands (de Lange et al., 2008). This functional coupling was suggested to reflect the interaction between motor cortical and visual areas while the rotation task was performed. Another example showed that frontal theta activity increased while posterior alpha activity was suppressed after button press responses in Go-noGo tasks (Mazaheri et al., 2009). Importantly the trial-by-trial anti-correlation between theta and alpha power was greatest after erroneous button press responses. This could reflect a top-down signal engaging the visual areas stronger in order to prevent subsequent errors.

In future work it will be important to apply these measures of cross-frequency interactions between gamma and alpha activity. In particular we predict a positive correlation between gamma power in task-relevant regions and alpha power in task-irrelevant regions. This correlation is expected to increase with task performance. Given the notion of pulsed inhibition we would also expect that alpha phase in one region will correlate with gamma power in another region. For instance, when a region is inhibited by alpha activity some processing can occur between the pulses of inhibition. This processing would have impact on down-stream regions and might be detected as gamma activity in the down-stream region phasically modulated by the alpha activity in sensory regions.

\section{LINKING GATING BY INHIBITIONTO THE BOLD SIGNAL}

Multiple studies combining fMRI and EEG have found that the BOLD signal correlates negatively with alpha activity (Goldman et al., 2002; Laufs et al., 2003; Feige et al., 2005; Scheeringa et al., 2009) whereas other studies have demonstrated a positive correlation between the BOLD signal and gamma activity (Logothetis et al., 2001; Niessing et al., 2005). These findings are consistent with the notion that alpha activity reflects functional inhibition. While most fMRI studies on cognitive processing focus on task-relevant regions, it would be interesting to investigate if BOLD decreases in task-irrelevant regions also are predictive of performance. This has been found in some studies. For instance, BOLD decreases in an extended network was shown to be predictive of memory formation (Daselaar et al., 2004). We propose to conduct further neuroimaging studies to investigate to what extend the deactivation of task-irrelevant regions reflected in a BOLD decrease correlates with performance. Are such decreases likewise correlated with an alpha increase? If it turns out to be a general phenomenon, it would suggest that our framework of gating by inhibition could be applied in fMRI studies as well.

\section{FUTURE RESEARCH QUESTIONS}

- While extensive work has been devoted to understanding the physiological mechanism generating for instance the gamma rhythm and spindle oscillations (Destexhe et al., 1998; Traub et al., 1999; Bartos et al., 2007; Mann and Paulsen, 2007; Cardin et al., 2009; Sohal et al., 2009) less is known about how the alpha rhythm is generated. While some models have been proposed (van Rotterdam et al., 1982; Stam et al., 1999; Jones et al., 2000, 2009; Suffczynski et al., 2001) the physiological underpinnings need to be better understood by intracranial investigations in humans and animals. These investigations should be complemented with computational modeling in order to understand synchronization properties and how receptor kinetics constrain the frequency of the oscillations.

- At what spatial scale does functional gating by alpha inhibition operate? This has implications for how general the principle of gating by alpha inhibition is. Interestingly it has been shown that the $10 \mathrm{~Hz}$ mu-rhythm is increased in the foot area of motor cortex when the hand area is engaged and vice versa (Pfurtscheller and Lopes da Silva, 1999; Suffczynski et al., 2001). Thus functional inhibition by alpha activity might operate within the primary motor cortex. However, the question of spatial scale is inherently difficult to assess with EEG and MEG due to volume conduction. A strong, but focal source will appear spatially distributed in the EEG/MEG sensors. ECoG data recorded from epileptic patients hold the promise of proving new insight into the issue of spatial scale (Lachaux et al., 2003; Miller et al., 2010).

- It is well established that the thalamus produces oscillatory activity in the alpha band and that is it linked to neocortical alpha generators (da Silva et al., 1973; Hughes and Crunelli, 2005; Lorincz et al., 2008, 2009). However, we need more insight into the role of the thalamus in the context of cognitive tasks. In particular, it would be important to employ tasks known to engage and disengage given neocortical areas and then investigate the thalamo-cortical coupling in the alpha band. This will also help to clarify if neocortical alpha oscillations are always a consequence of a thalamic drive or if they can be generated independently.

- We have proposed that information flow is directed by disengaging task-irrelevant regions by alpha activity. The hypothesis is derived from EEG and MEG studies in which alpha activity is characterized in sensory areas. Is the gating by alpha inhibition only constrained to sensory areas or does is generalize to higher order regions as well? This is a difficult question to answer by EEG and MEG alone since these techniques in particular seem to be sensitive to oscillatory activity produced in extended sensory and motor regions (Hari and Salmelin, 1997). To effectively answer this question we need to apply intracranial recordings in humans within the context of cognitive tasks. As intracranial data are becoming increasingly available from presurgical investigation in epileptic patients, we expect to be able to address this issue in the near future.

- Do rhythms in other frequency bands reflect active processing or functional inhibition? With respect to beta band activity several studies point to this rhythm reflecting functional 
inhibition of motor cortical regions (Hari and Salmelin, 1997; Jensen et al., 2005; Miller et al., 2010). However, beta band activity has also been proposed to play a more active role such as maintenance of the status quo (Engel and Fries, 2010). Theta activity has been assigned an activity processing role for instance in the hippocampus (Kahana et al., 2001; Jensen and Lisman, 2005; Buzsaki, 2006). However, it should be mentioned that rat hippocampal theta activity is a consequence of both a tonic cholinergic input and a phasic GABAergic drive from the medial septum. This phasic GABAergic drive will provide pulses inhibition (Vertes and Kocsis, 1997). Finally, the low frequency delta rhythm has been proposed to set the excitability of a given region. The phase adjustment of the delta rhythm might be important for anticipatory processes (Schroeder and Lakatos, 2009). From this perspective the phase of the delta rhythm plays an active role in controlling neuronal processing. The functional role of these oscillations can be assessed by developing and using tasks which address how the engagement and disengagement of specific regions are reflected in various frequency bands.

- How do top-down mechanisms control the alpha activity? One possibility is that frontal executive functions reflected in the theta band serve to exercise the top-down control. Support for this notion has been found in recent studies employing Go-noGo tasks (Mazaheri et al., 2009, 2010). In order to identify the areas involved in top-down control we need to apply multi-modal techniques such as TMS combined with EEG. For instance it was recently shown that perturbing the right IPS and FEF with TMS modulated the posterior alpha activity (Capotosto et al., 2009). Another possibly is to combine fMRI and EEG in order to identify frontal regions from which the BOLD signal correlates with posterior alpha activity.

- While the posterior alpha activity clearly is modulated by topdown control there are also competitive mechanisms of a more local nature at play. For instance there is evidence for inter-hemispheric inhibition in context of visual attention (Kinsbourne, 1977). This is also supported by monkey data in which the ipsilateral primary somatosensory cortex is inhibited by sensory

\section{REFERENCES}

Babiloni, C., Vecchio, F., Bultrini, A., Luca Romani, G., and Rossini, P. M. (2006). Pre- and poststimulus alpha rhythms are related to conscious visual perception: a high-resolution EEG study. Cereb. Cortex 16, 1690-1700.

Bahramisharif,A., van Gerven, M., Heskes, T., and Jensen, O. (2010). Covert attention allows for continuous control of brain-computer interfaces. Eur. J. Neurosci. 31, 1501-1508.

Bartos, M., Vida, I., and Jonas, P. (2007). Synaptic mechanisms of synchronized gamma oscillations in inhibitory interneuron networks. Nat. Rev. Neurosci. 8, 45-56.

Berger, H. (1929). Über das elektroenkephalogramm des menschen. Arch. Psychiatr. Nervenkr. 87, 527-570.
Bressler, S. L. (1995). Large-scale cortical networks and cognition. Brain Res. Brain Res. Rev. 20, 288-304.

Bruns, A., Eckhorn, R., Jokeit, H., and Ebner, A. (2000). Amplitude envelope correlation detects coupling among incoherent brain signals. Neuroreport 11, 1509-1514.

Busch, N. A., Dubois, J., and VanRullen, R. (2009). The phase of ongoing EEG oscillations predicts visual perception. J. Neurosci. 29, 7869-7876.

Buzsaki, G. (2006). Rhythms of the Brain. Oxford; New York: Oxford University Press.

Capotosto, P., Babiloni, C., Romani, G. L., and Corbetta, M. (2009). Frontoparietal cortex controls spatial attention through modulation of anticipatory alpha rhythms. J. Neurosci. 29, 5863-5872.

stimulation (Lipton et al., 2006). Such inter-hemispheric inhibition could have a direct consequence for the control of the alpha activity (Thut et al., 2006). Interestingly, it was shown that engaging the motorcortical hand area result in a alpha and beta band response in foot areas (Pfurtscheller et al., 2000). This suggests that competition between motor cortical areas is reflected in oscillatory brain activity within the motor strip.

- Several disorders are related to problems in attentional control. One of the key symptoms of ADHD is the reduced ability to suppress distracting input (Doyle, 2006). Could this be reflected by an inability to control the posterior alpha activity and thus a failure in the gating by inhibition mechanism? It was recently demonstrated that during an attentional cuing task, children with ADHD were not able to modulate their alpha activity to prepare for the upcoming stimuli. In contrast, children from the control group performing the same task showed pre-stimulus alpha changes in the sensory systems which were anti-correlated with theta from the frontal control regions (Mazaheri et al., 2010).

\section{CONCLUSION}

We have presented a working hypothesis postulating that information is gated through the brain by functional inhibition of taskirrelevant areas. Further, the functional inhibition is reflected by oscillatory activity in the alpha band. Our hypothesis provides a general framework for understanding how the functional architecture of the brain is shaped. Our hypothesis provides a putative explanation for why the alpha activity is the strongest electrophysiological signal measured from the human brain: optimal performance requires an effective inhibition of task-irrelevant regions. While several studies support this framework more empirical work is required in order to substantiate the principle of gating by inhibition.

\section{ACKNOWLEDGMENTS}

This research was supported by The Netherlands Organization for Scientific Research (NWO): Innovational Research Incentive Schemes, VICI grant number: 453-09-002; ALW Open Competition, grant number 817.02.010; and a Rubicon grant.

Cardin, J. A., Carlen, M., Meletis, K. Knoblich, U., Zhang, F., Deisseroth, K., Tsai, L. H., and Moore, C. I. (2009). Driving fast-spiking cells induces gamma rhythm and controls sensory responses. Nature 459, 663-667.

Colgin, L. L., Denninger, T., Fyhn, M., Hafting, T., Bonnevie, T., Jensen, O., Moser, M. B., and Moser, E. I. (2009). Frequency of gamma oscillations routes flow of information in the hippocampus. Nature 462, 353-357.

Crone, N. E., Sinai, A., and Korzeniewska, A. (2006). High-frequency gamma oscillations and human brain mapping with electrocorticography. Prog. Brain Res. 159, 275-295.

da Silva, F. H., van Lierop, T. H., Schrijer, C. F., and van Leeuwen, W. S. (1973). Organization of thalamic and cortical alpha rhythms: spectra and coherences. Electroencephalogr. Clin. Neurophysiol. 35, 627-639.

Daselaar, S.M.,Prince, S.E., and Cabeza, R (2004). When less means more: deactivations during encoding that predict subsequent memory. Neuroimage 23, 921-927.

de Lange, F. P., Jensen, O., Bauer, M., and Toni, I. (2008). Interactions between posterior gamma and frontal alpha/ beta oscillations during imagined actions. Front. Hum. Neurosci. 2:7. doi: 10.3389/neuro.09.007.2008.

Del Percio, C., Babiloni, C., Bertollo, M., Marzano, N., Iacoboni, M. Infarinato, F., Lizio, R., Stocchi, M., Robazza, C., Cibelli, G., Comani, S., and Eusebi, F. (2009). Visuoattentional and sensorimotor alpha 
rhythms are related to visuo-motor performance in athletes. Hum. Brain Mapp. 30, 3527-3540.

Destexhe, A., Contreras, D., and Steriade, M. (1998). Mechanisms underlying the synchronizing action of corticothalamic feedback through inhibition of thalamic relay cells. J. Neurophysiol. 79, 999-1016.

Doyle, A. E. (2006). Executive functions in attention-deficit/hyperactivity disorder. J. Clin. Psychiatry 67(Suppl. 8), 21-26.

Engel, A. K., and Fries, P. (2010). Betaband oscillations-signalling the status quo? Curr. Opin. Neurobiol. 20, 156-65.

Fanselow, E. E., Richardson, K. A., and Connors, B.W. (2008). Selective, statedependent activation of somatostatinexpressing inhibitory interneurons in mouse neocortex. J. Neurophysiol. 100 2640-2652.

Feige, B., Scheffler, K., Esposito, F., Di Salle, F., Hennig, J., and Seifritz, E. (2005). Cortical and subcortical correlates of electroencephalographic alpha rhythm modulation. J. Neurophysiol. 93, 2864-2872.

Fries, P. (2005). A mechanism for cognitive dynamics: neuronal communication through neuronal coherence. Trends Cogn. Sci. (Regul. Ed.) 9, 474-480.

Fries, P., Nikolic, D., and Singer,W. (2007). The gamma cycle. Trends Neurosci. 30 , 309-316.

Friston, K. J., Buechel, C., Fink, G. R., Morris, J., Rolls, E., and Dolan, R. J. (1997). Psychophysiological and modulatory interactions in neuroimaging. Neuroimage 6, 218-229.

Fu,K.M.,Foxe,J.J., Murray,M.M.,Higgins, B. A., Javitt, D. C., and Schroeder, C. E. (2001). Attention-dependent suppression of distracter visual input can be cross-modally cued as indexed by anticipatory parieto-occipital alphaband oscillations. Brain Res. Cogn. Brain Res. 12, 145-152.

Goldman, R. I., Stern, J. M., Engel, J. Jr., and Cohen, M.S. (2002). Simultaneous EEG and fMRI of the alpha rhythm. Neuroreport 13, 2487-2492.

Grimault, S., Robitaille, N., Grova, C., Lina, J. M., Dubarry, A. S., and Jolicoeur, P. (2009). Oscillatory activity in parietal and dorsolateral prefrontal cortex during retention in visual short-term memory: additive effects of spatial attention and memory load. Hum. Brain Mapp. 30, 3378-3392.

Haegens, S., Osipova, D., Oostenveld, R., and Jensen, O. (2010). Somatosensory working memory performance in humans depends on both engagement and disengagement of regions in a distributed network. Hum. Brain Mapp. 31, 26-35.
Handel, B., Haarmeier, T, and Jensen, O. (2010). Alpha oscillations correlate with the successful inhibition of unattended stimuli. J. Cogn. Neurosci. doi: 10.1162/jocn.2010.21557

Hanslmayr, S., Aslan, A., Staudigl, T. Klimesch, W., Herrmann, C. S., and Bauml, K. H. (2007). Prestimulus oscillations predict visual perception performance between and within subjects. Neuroimage 37, 1465-1473.

Hari, R., and Salmelin, R. (1997). Human cortical oscillations: a neuromagnetic view through the skull. Trends Neurosci. 20, 44-49.

Hughes, S. W., and Crunelli, V. (2005) Thalamic mechanisms of EEG alpha rhythms and their pathological implications. Neuroscientist 11, 357-372.

Jensen, O., and Colgin, L. L. (2007). Crossfrequency coupling between neuronal oscillations. Trends Cogn. Sci. (Regul. Ed.) 11,267-269.

Jensen, O., Gelfand, J., Kounios, J., and Lisman, J. E. (2002). Oscillations in the alpha band $(9-12 \mathrm{~Hz})$ increase with memory load during retention in a short-term memory task. Cereb. Cortex 12, 877-882.

Jensen, O., Goel, P., Kopell, N., Pohja, M. Hari, R., and Ermentrout, B. (2005). On the human sensorimotor-cortex beta rhythm: sources and modeling. Neuroimage 26, 347-355.

Jensen, O., Kaiser, J., and Lachaux, J. P. (2007). Human gamma-frequency oscillations associated with attention and memory. Trends Neurosci. 30, 317-324.

Jensen, O., and Lisman, J. E. (2005) Hippocampal sequence-encoding driven by a cortical multi-item working memory buffer. Trends Neurosci. $28,67-72$.

Jerbi, K., Ossandon, T., Hamame, C. M., Senova, S., Dalal, S. S., Jung, J., Minotti, L., Bertrand, O., Berthoz, A., Kahane, P., and Lachaux, J. P. (2009). Task-related gamma-band dynamics from an intracerebral perspective: review and implications for surface EEG and MEG. Hum. Brain Mapp. 30, 1758-1771.

Jokisch, D., and Jensen, O. (2007) Modulation of gamma and alpha activity during a working memory task engaging the dorsal or ventral stream. J. Neurosci. 27, 3244-3251.

Jones, S. R., Pinto, D. J., Kaper, T. J., and Kopell, N. (2000). Alpha-frequency rhythms desynchronize over long cortical distances: a modeling study. J. Comput. Neurosci. 9, 271-291.

Jones, S. R., Pritchett, D. L., Sikora, M. A. Stufflebeam, S. M., Hamalainen, M., and Moore, C. I. (2009). Quantitative analysis and biophysically realistic neural modeling of the MEG mu rhythm: rhythmogenesis and modulation of sensory-evoked responses. $J$. Neurophysiol. 102, 3554-3572.

Kahana, M. J., Seelig, D., and Madsen, J. R. (2001). Theta returns. Curr. Opin. Neurobiol. 11, 739-744.

Kaiser, J., and Lutzenberger, W. (2005) Cortical oscillatory activity and the dynamics of auditory memory processing. Rev. Neurosci. 16, 239-254.

Kelly, S. P., Gomez-Ramirez, M., and Foxe, J. J. (2009). The strength of anticipatory spatial biasing predicts target discrimination at attended locations: a high-density EEG study. Eur. J. Neurosci. 30, 2224-2234.

Kerlin, J. R., Shahin, A. J., and Miller, L. M. (2010). Attentional gain control of ongoing cortical speech representations in a "cocktail party". J. Neurosci. 30, 620-628.

Kinsbourne, M. (1977). Hemi-neglect and hemisphere rivalry. Adv. Neurol. $18,41-49$.

Klimesch, W., Sauseng, P., and Hanslmayr S. (2007). EEG alpha oscillations: the inhibition-timing hypothesis. Brain Res. Brain Res. Rev. 53, 63-88.

Konig,P.,Engel,A. K., and Singer,W. (1996) Integrator or coincidence detector? The role of the cortical neuron revisited. Trends Neurosci. 19, 130-137.

Lachaux, J. P., Rudrauf, D., and Kahane, P. (2003). Intracranial EEG and human brain mapping. J. Physiol. Paris 97 613-628.

Laufs, H., Kleinschmidt, A., Beyerle, A. Eger, E., Salek-Haddadi, A., Preibisch, C., and Krakow, K. (2003). EEGcorrelated fMRI of human alpha activity. Neuroimage 19, 1463-1476.

Lehtela, L., Salmelin, R., and Hari, R. (1997). Evidence for reactive magnetic $10-\mathrm{Hz}$ rhythm in the human auditory cortex. Neurosci. Lett. 222, 111-114.

Linkenkaer-Hansen, K., Nikulin, V. V. Palva, S., Ilmoniemi, R. J., and Palva J. M. (2004). Prestimulus oscillations enhance psychophysical performance in humans. J. Neurosci. 24 10186-10190.

Lipton, M. L., Fu, K. M., Branch, C. A. and Schroeder, C. E. (2006). Ipsilateral hand input to area $3 \mathrm{~b}$ revealed by converging hemodynamic and electrophysiological analyses in macaque monkeys. J. Neurosci. 26, 180-185.

Logothetis, N. K., Pauls, J., Augath, M. Trinath, T., and Oeltermann, A. (2001). Neurophysiological investigation of the basis of the fMRI signal. Nature 412, 150-157.

Lorincz, M. L., Crunelli, V., and Hughes, S. W. (2008). Cellular dynamics of cholinergically induced alpha $(8-13 \mathrm{~Hz})$ rhythms in sensory thalamic nuclei in vitro. J. Neurosci. 28 660-671.
Lorincz, M. L., Kekesi, K. A., Juhasz, G., Crunelli, V., and Hughes, S. W. (2009). Temporal framing of thalamic relaymode firing by phasic inhibition during the alpha rhythm. Neuron 63 683-696.

Mann, E. O., and Paulsen, O. (2007). Role of GABAergic inhibition in hippocampal network oscillations. Trends Neurosci. 30, 343-349.

Mathewson, K. E., Gratton, G., Fabiani, M., Beck, D. M., and Ro, T. (2009). To see or not to see: prestimulus alpha phase predicts visual awareness. $J$. Neurosci. 29, 2725-2732.

Mazaheri,A., Coffey-Corina, S., Mangun, G. R., Bekker, E. M., Berry, A. S., and Corbett, B. A. (2010). Functional disconnection of frontal cortex and visual cortex in attention-deficit/ hyperactivity disorder. Biol. Psychiatry 67, 617-623.

Mazaheri, A., and Jensen, O. (2008). Asymmetric amplitude modulations of brain oscillations generate slow evoked responses. J. Neurosci. 28 7781-7787.

Mazaheri, A., and Jensen, O. (2010). Rhythmic pulsing: linking ongoing brain activity with evoked responses. Front. Hum. Neurosci. 4:177. doi: 10.3389/fnhum.2010.00177

Mazaheri, A., Nieuwenhuis, I. L., van Dijk, H., and Jensen, O. (2009). Prestimulus alpha and mu activity predicts failure to inhibit motor responses. Hum. Brain Mapp. 30, 1791-800.

Meeuwissen,E.B., Takashima,A.,Fernandez $\mathrm{G}$., and Jensen, $\mathrm{O}$. (in press). Increase in posterior alpha activity during rehearsal predicts successful long-term memory formation of word sequences. Hum. Brain Mapp.

Miller, K. J., Schalk, G., Fetz, E. E., den Nijs, M., Ojemann, J. G., and Rao, R. P. (2010). Cortical activity during motor execution, motor imagery, and imagery-based online feedback. Proc. Natl. Acad. Sci. U.S.A. 107, 4430-4435

Niedermeyer, E., and Lopes da Silva, F. H. (1999). Electroencephalography: Basic Principles, Clinical Applications, and Related Fields, 4th Edn. Baltimore: Williams and Wilkins

Niessing, J., Ebisch, B., Schmidt, K. E. Niessing, M., Singer, W., and Galuske, R. A. (2005). Hemodynamic signals correlate tightly with synchronized gamma oscillations. Science 309, 948-951.

Nikulin, V. V., Linkenkaer-Hansen, K., Nolte, G., Lemm, S., Muller, K. R., Ilmoniemi, R. J., and Curio, G. (2007).A novel mechanism for evoked responses in the human brain. Eur. J. Neurosci. 25, 3146-3154.

Osipova, D., Hermes, D., and Jensen, O. (2008). Gamma power is phaselocked to posterior alpha activity. $P L O S$ 
ONE 3, e3990. doi: 10.1371/journal. pone. 0003990 .

Paller, K. A., Kutas, M., and Mayes, A. R. (1987). Neural correlates of encoding in an incidental learning paradigm. Electroencephalogr. Clin. Neurophysiol. 67, 360-371.

Palva, J. M., Monto, S., Kulashekhar, S., and Palva, S. (2010). Neuronal synchrony reveals working memory networks and predicts individual memory capacity. Proc. Natl. Acad. Sci. U.S.A. 107, 7580-7585.

Palva, J. M., Palva, S., and Kaila, K. (2005). Phase synchrony among neuronal oscillations in the human cortex. $J$. Neurosci. 25, 3962-3972.

Palva, S., and Palva, J. M. (2007). New vistas for alpha-frequency band oscillations. Trends Neurosci. 30, 150-158.

Pfurtscheller, G. (1992). Event-related synchronization (ERS): an electrophysiological correlate of cortical areas at rest. Electroencephalogr. Clin. Neurophysiol. 83, 62-69.

Pfurtscheller, G., and Lopes da Silva, F. H. (1999). Event-related EEG/MEG synchronization and desynchronization: basic principles. Clin. Neurophysiol. 110, 1842-1857.

Pfurtscheller, G., Neuper, C., PichlerZalaudek, K., Edlinger, G., and Lopes da Silva, F. H. (2000). Do brain oscillations of different frequencies indicate interaction between cortical areas in humans? Neurosci. Lett. 286, 66-68.

Pfurtscheller, G., Stancak, A. Jr., and Neuper, C. (1996). Event-related synchronization (ERS) in the alpha band-an electrophysiological correlate of cortical idling: a review. Int. J. Psychophysiol. 24, 39-46.

Rajagovindan, R., and Ding, M. (2010). From prestimulus alpha oscillation to visual-evoked response: an inverted-u function and its attentional modulation. J. Cogn. Neurosci. doi:10.1162/ jocn.2010.21478

Rihs, T. A., Michel, C. M., and Thut, G. (2007). Mechanisms of selective inhibition in visual spatial attention are indexed by alpha-band EEG synchronization. Eur. J. Neurosci. 25, 603-610.

Romei, V., Brodbeck, V., Michel, C., Amedi, A., Pascual-Leone, A., and Thut, G. (2008). Spontaneous fluctuations in posterior alpha-band EEG activity reflect variability in excitability of human visual areas. Cereb. Cortex 18, 2010-2018.

Sauseng, P., Klimesch, W., Heise, K. F., Gruber, W. R., Holz, E., Karim, A. A., Glennon, M., Gerloff, C., Birbaumer, N., and Hummel, F. C. (2009). Brain oscillatory substrates of visual shortterm memory capacity. Curr. Biol. 19, 1846-1852.

Scheeringa, R., Petersson, K. M., Oostenveld, R., Norris, D. G., Hagoort, P., and Bastiaansen, M. C. (2009). Trialby-trial coupling between EEG and BOLD identifies networks related to alpha and theta EEG power increases during working memory maintenance. Neuroimage 44, 1224-1238.

Schroeder, C. E., and Lakatos, P. (2009). Low-frequency neuronal oscillations as instruments of sensory selection. Trends Neurosci. 32, 9-18.

Schubert, R., Haufe, S., Blankenburg, F., Villringer, A., and Curio, G. (2009). Now you'll feel it, now you won't: EEG rhythms predict the effectiveness of perceptual masking. J. Cogn. Neurosci. 21, 2407-2419.

Sohal, V. S., Zhang, F., Yizhar, O., and Deisseroth, K. (2009). Parvalbumin neurons and gamma rhythms enhance cortical circuit performance. Nature 459, 698-702.

Stam, C. J., Pijn, J. P., Suffczynski, P., and Lopes da Silva, F. H. (1999). Dynamics of the human alpha rhythm: evidence for non-linearity? Clin. Neurophysiol. 110, 1801-1813.

Suffczynski, P., Kalitzin, S., Pfurtscheller G., and Lopes da Silva, F. H. (2001) Computational model of thalamocortical networks: dynamical control of alpha rhythms in relation to focal attention. Int. J. Psychophysiol. 43, 25-40.

Thut, G., and Miniussi, C. (2009). New insights into rhythmic brain activity from TMS-EEG studies. Trends Cogn. Sci. (Regul. Ed.) 13, 182-189.

Thut, G., Nietzel, A., Brandt, S. A., and Pascual-Leone, A. (2006). Alpha-band electroencephalographic activity over occipital cortex indexes visuospatial attention bias and predicts visual target detection. J. Neurosci. 26, 9494-9502.
Traub, R. D., Jefferys, J. G. R., and Whittington, M. A. (1999). Fast Oscillations in Cortical Circuits. Cambridge, MA: MIT Press.

Tuladhar,A.M., ter Huurne, N., Schoffelen, J. M., Maris, E., Oostenveld, R., and Jensen, O. (2007). Parieto-occipital sources account for the increase in alpha activity with working memory load. Hum. Brain Mapp. 28, 785-792.

Van Der Werf, J., Jensen, O., Fries, P., and Medendorp, W. P. (2008). Gammaband activity in human posterior parietal cortex encodes the motor goal during delayed prosaccades and antisaccades. J. Neurosci. 28 8397-8405.

van Dijk, H., Nieuwenhuis, I., Schulte, M. and Jensen, O. (2010) Left temporal alpha band activity increases during working memory retention of pitches. Eur. J. Neurosci. 31, 1701-1707.

van Dijk, H., Schoffelen, J. M., Oostenveld, R., and Jensen, O. (2008). Prestimulus oscillatory activity in the alpha band predicts visual discrimination ability. J. Neurosci. 28, 1816-1823.

van Dijk, H., van der Werf, J., Mazaheri, A., Medendorp, W. P., and Jensen, O. (2010). Modulations in oscillatory activity with amplitude asymmetry can produce cognitively relevant event-related responses. Proc. Natl. Acad. Sci. U.S.A. 107, 900-905.

van Gerven, M., and Jensen, O. (2009) Attention modulations of posterior alpha as a control signal for two-dimensional brain-computer interfaces. J. Neurosci. Methods 179, 78-84.

van Rotterdam, A., Lopes da Silva, F. H., van den Ende, J., Viergever, M. A. and Hermans, A. J. (1982). A model of the spatial-temporal characteristics of the alpha rhythm. Bull. Math. Biol. 44, 283-305.

VanRullen, R., and Koch, C. (2003). Is perception discrete or continuous? Trends Cogn. Sci. 7, 207-213.

Varela, F., Lachaux, J.P., Rodriguez, E., and Martinerie, J. (2001). The brainweb: phase synchronization and largescale integration. Nat. Rev. Neurosci. 2, 229-239.

Varela, F. J., Toro, A., John, E. R., and Schwartz, E. L. (1981). Perceptual framing and cortical alpha rhythm. Neuropsychologia 19, 675-686.
Vertes, R. P., and Kocsis, B. (1997). B rainstem-dien cephaloseptohippocampal systems controlling the theta rhythm of the hippocampus. Neuroscience 81, 893-926.

Voytek, B., Canolty, R. T., Shestyuk, A., Crone, N., Parvizi, J., and Knight, R. T. (2010). Shifts in gamma phaseamplitude coupling frequency from theta to alpha over posterior cortex during visual tasks. Front. Hum. Neurosci. 4:191. doi: 10.3389/ fnhum.2010.00191.

Womelsdorf, T., and Fries, P. (2007). The role of neuronal synchronization in selective attention. Curr. Opin. Neurobiol. 17, 154-160.

Worden, M. S., Foxe, J. J., Wang, N., and Simpson, G. V. (2000). Anticipatory biasing of visuospatial attention indexed by retinotopically specific alpha-band electroencephalography increases over occipital cortex. J. Neurosci. 20, RC63.

Zhang, Y., and Ding, M. (2010). Detection of a weak somatosensory stimulus: role of the prestimulus mu rhythm and its top-down modulation. J. Cogn. Neurosci. 22, 307-322.

Zhang, Y., Wang, X., Bressler, S. L., Chen, Y., and Ding, M. (2008). Prestimulus cortical activity is correlated with speed of visuomotor processing. $J$ Cogn. Neurosci. 20, 1915-1925.

Conflict of Interest Statement: The authors declare that the research was conducted in the absence of any commercial or financial relationships that could be construed as a potential conflict of interest.

Received: 01 June 2010; paper pending published: 05 July 2010; accepted: 14 September 2010; published online: 04 November 2010.

Citation: Jensen O and Mazaheri A (2010) Shaping functional architecture by oscillatory alpha activity: gating by inhibition. Front. Hum. Neurosci. 4:186. doi: 10.3389/ fnhum.2010.00186

Copyright (c) 2010 Jensen and Mazaheri. This is an open-access article subject to an exclusive license agreement between the authors and the Frontiers Research Foundation, which permits unrestricted use, distribution, and reproduction in any medium, provided the original authors and source are credited. 